\title{
PROBLEMAS DESCRIPTIVOS Y PENSAMIENTO NUMÉRICO: EL CASO DE LAS CIEN AVES DE CORRAL
}

\begin{abstract}
Bernardo Gómez Alfonso
Bajo el marco metodológico del análisis didáctico y del análisis históricoepistemológico se presenta un estudio sobre los problemas descriptivos clásicos que emanan del de "las cien aves de corral". Se presenta una aproximación global a su larga evolución histórica como objeto de enseñanza, se da cuenta de algunas de sus lecturas analiticas, aritméticas y cartesianas, y se analizan sus métodos y reglas de resolución.
\end{abstract}

Términos clave: Análisis didáctico; Análisis histórico-epistemológico; Libros de texto; Problemas de mezclas; Reglas y métodos

Descriptive Problems and Numerical Thinking: The Case of one Hundred Poultry

Under the methodological framework of the didactic analysis and historicalepistemological analysis a study of the classical descriptive problems that emanate from the "hundred poultry" is presented. An integrated approach is presented to its long historical evolution as an object of teaching, he realizes some of its analytical, arithmetic and reading cartesian, and its methods and resolution rules are analyzed.

Keywords: Didactic analysis; Historical-epistemological analysis; Mixing problems; Rules and methods; Textbooks

Los problemas aritméticos de enunciado verbal que ha transmitido la tradición recogida en los libros de enseñanza se suelen introducir en el aula con diferentes propósitos. A saber: para modelar las operaciones elementales, como es el caso de los problemas aditivos y multiplicativos; para dar respuesta a necesidades humanas, como ocurre con los problemas mercantiles; y, para desafiar el ingenio matemático, como se hace con los problemas recreativos con los que se pretende mostrar que las matemáticas también son una actividad intelectual pura.

Gómez, B. (2016). Problemas descriptivos y pensamiento numérico: el caso de las cien aves de corral. PNA, 10(3), 218-241. 
Remontándose a las antiguas culturas matemáticas se encuentra un grupo de problemas descriptivos, llamados así porque narran una historia o describen una situación (Swetz, 2014), que aunque se presentaban con escenarios o contextos pseudorealistas y generaban respuestas que no eran prácticas, dieron lugar a verdaderos problemas prácticos.

El enunciado de estos problemas ha evolucionado al compás de las cambiantes necesidades sociales, educativas y económicas, actualizándose y adaptándose a los tiempos, pero conservando su contenido matemático.

El capitalismo mercantil europeo a finales de la Edad Media vino acompañado por un auge en la computación numérica y la resolución de problemas. Entre los siglos XII al XV se escribieron manuscritos, y libros impresos ${ }^{1}$ que, además de promover la enseñanza de las matemáticas comerciales, incorporaron colecciones de problemas. Estos libros no solo desempeñaron un papel destacado en la introducción de los numerales indo-arábigos y sus correspondientes algoritmos, sino también en la estandarización de los problemas de índole mercantil ${ }^{2}$ y de algunos otros problemas de carácter recreativo, que bajo la forma de problema tipo o estereotipo los hacen fácilmente reconocibles.

Esto hizo que la aritmética elemental se configurara con una parte final o capítulo formado por una variada colección de métodos para resolver problemas particulares; por ejemplo, la regla de tres simple y compuesta, la regla del interés u otros métodos que se describen a continuación.

\section{MÉTODOS PARA RESOLVER PROBLEMAS}

Los métodos que se describen en este apartado son siete: el método de inversión, la regla de posición y remoción, las reglas de falsa posición, la regla de dos, regla de aligación, regla de la cadena y la regla de compañías.

\section{Método de inversión}

Este método aritmético consiste en volver hacia atrás, desandando el camino recorrido. Paso a paso las operaciones dadas se transforman en operaciones inversas. En Aryabhatiya de Aryabhata (476 d. C.), el método se introduce diciendo: multiplicadores se vuelven divisores y divisores se vuelven multiplicadores, aditivos se vuelven sustractivos y sustractivos se vuelven aditivos (ver Shen, Crossley y Lun,

\footnotetext{
${ }^{1}$ Las primeras aritméticas impresas fueron: en Italia, La Aritmética de Treviso en 1478; en Alemania, Rechenbuch (libro de cálculo), de Ulrich Wagner; y el Behende und hübsche Rechenung auff allen Kauffmanschafft de J. Widman en Leipzig en 1489. Incluye por primera vez los signos + y -. En España, La Suma de la art de arismetica de Frances Santcliment en 1482. En Inglaterra, De arte supputande libri quattuor de Cuthbert Tunstall en 1522.

${ }^{2}$ En el primer libro impreso de aritmética, la Aritmética de Treviso (1478), se incluyeron 62 problemas que cubrían diferentes cuestiones mercantiles: cálculo de intereses, pérdidas y ganancias, seguros, trueques, compañías o sociedades mercantiles, cálculo de taras y sobrepeso, pago de impuestos y aduanas, tiempos y coste del transporte, aligaciones y otros problemas diversos.
} 
1999, p. 384). En al-Amuli (1547-1622), "este procedimiento consiste en hacer lo contrario de lo que propone el enunciado; pide doblar, se semisuma; pide sumar, se resta; pide raíz, se cuadra, etc.; comenzando por la última parte del problema se obtiene la solución" (Meavilla, 2000, p. 81).

\section{Regla de posición y remoción}

La "posición y remoción" quiere decir propiamente poner y quitar. Y esto es según dice la regla general, que cuando encuentras más de lo que quieres, debes quitar de lo que hace subir y debes aumentar de lo que hace descender. Y, por el contrario, cuando se tiene menos de lo que se quiere, lo debemos quitar de lo que hace bajar y poner en lo que hace crecer. Por declaración de esta regla has de saber que no es propiamente una regla, sino un consejo (SantCliment, 1482/1998, p. 328).

\section{Reglas de falsa posición}

Los chinos del primer milenio de nuestra era desarrollaron un método que ha estado presente en los libros de texto de aritmética y de álgebra elemental hasta los comienzos del siglo XX. Su nombre, falsa posición, está asociado al proceso algorítmico que encierra, ya que para obtener la solución hay que tomar valores supuestos, y por lo tanto falsos, en el lugar de la incógnita. Con ellos se hacen los cálculos que se señalan en el enunciado del problema para obtener resultados erróneos. Con estos datos, los supuestos y los erróneos, la aplicación de la regla permite obtener el resultado correcto. En realidad, hay dos reglas, según que sean necesarios uno o dos valores supuestos, llamadas: de una o de dos falsas posiciones.

El fundamento de la regla de una falsa posición liga las nociones proporcionalidad, ecuaciones de primer grado y función lineal. Las condiciones del enunciado se pueden modelizar con una expresión lineal como esta: $y=a_{1} x+a_{2} x+a_{3} x+\ldots+a_{n} x$; siendo $a_{i}$ un número entero o fraccionario, lo que equivale a $y=\left(a_{1}+a_{2}+a_{3}+\ldots+a_{n}\right) x$ o, poniendo $a$ en vez de $a_{1}+a_{2}+a_{3}+\ldots+a_{n}$, $y=a x$. Desde este enfoque, lo que pide el problema es determinar la abcisa $x$ de un punto $(x, y)$, cuando se conoce tanto su ordenada $y$ como el coeficiente $a$ de la función. Para obtener el valor buscado la regla dice que se determine otro punto $\left(x_{1}, y_{1}\right)$, para ello se toma como abcisa un valor supuesto $x_{1} \mathrm{y}$ se calcula su correspondiente valor en la función, $y_{1}=a x_{1}$. Aplicando al par de valores $(x, y)$, $\left(x_{1}, y_{1}\right)$ la idea básica de la linealidad se obtiene la proporción $\frac{x}{y}=\frac{x_{1}}{y_{1}}$ que da la fórmula $x=y \cdot \frac{x_{1}}{y_{1}}$ cuyo enunciado es la famosa regla.

También se pueden interpretar como una ecuación de primer grado con una incógnita: $b=a x$. La regla manda que se resuelva la ecuación dando un valor supuesto a $x=x_{1}$, que da lugar al error $b_{1}, b_{1}=a x_{1}$. Resolviendo el sistema formado 
por las dos ecuaciones: $b=a x$ y $b_{1}=a x_{1}$, se obtiene una igualdad, que no es otra cosa que la proporción anterior, de la cual se sigue el valor de $x$. Obsérvese que en esta regla, la elección del valor supuesto es fundamental. Por ejemplo, tomando como valor supuesto $x_{1}=1, y_{1}=a$, entonces $x=\frac{y}{a}$, que es la forma actual de resolver estos problemas.

\section{Regla de dos}

Con este nombre se conocía en las aritméticas medievales y renacentistas una regla para resolver los problemas de acciones simultáneas (móviles, grifos, trabajos en conjunto) que se formulaba como la división de un producto por una diferencia o una suma, según que el problema sea de alcanzar o de encontrar.

\section{Regla de aligación}

Bajo el nombre de regla de aligación se conoce el procedimiento general para resolver los problemas relacionados con las mezclas y aleaciones. Por mezcla se entiende la unión de varias sustancias de distinta calidad y precio; si son metales se llama aleación, y si son líquidos y sustancias, como por ejemplo la mezcla de agua y sal se llama disolución.

\section{Regla de la cadena}

La regla de la cadena se ilustra en el esquema gráfico que resuelve el siguiente problema (figura 1).

Si 39 reales de Valencia son 56 de Barcelona, y 14 de Barcelona son 8 de Aragón, y 16 de Aragón son 30 de Castilla; pídese ¿100 reales de Castilla cuántos serán de Valencia?

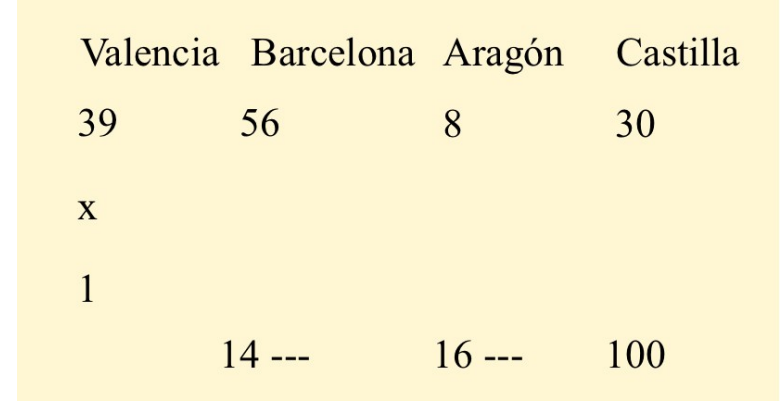

Figura 1. Esquema gráfico de resolución

Hecho esto, multiplíquense los números unos por otros, según lo señalan las líneas, esto es $39 \cdot 14 \cdot 16 \cdot 100$ y el producto 873600 , será el dividendo, porque en él está incluido el número 100, que tiene anexa la cuestión. Multiplíquese así mismo entre sí $56 \cdot 8 \cdot 30$ y el producto 13440 , será el partidor. Hecha la división hallaremos 65 reales (Corachán, 1699, pp. 249-250). 


\section{Regla de compañías}

Tiene por objeto el reparto proporcional de los beneficios o pérdidas habidos en un negocio entre las personas que han intervenido con sus capitales.

Hasta el siglo XIX, los problemas descriptivos eran una parte principal de la enseñanza de las matemáticas, y se consideraban la culminación del conocimiento aritmético. Sin embargo, según fue cambiando el modelo de enseñanza y el contenido de los libros de texto de matemáticas, fue disminuyendo la confianza en el poder educativo de estos problemas, hasta el punto de que muchos de ellos han desaparecido de los libros de texto actuales, o han quedado reducidos a mero entretenimiento. Claro está que desapareció el interés por algunos de los temas y algunos de los métodos especiales que, como el de falsa posición, por ejemplo, fueron ventajosamente sustituidos por las ecuaciones lineales.

El hecho de que muchos de los problemas descriptivos se pueden resolver con facilidad y de modo general con el método cartesiano ha contribuido a perder de vista que estos problemas han servido para comunicar los usos, técnicas y razonamientos de las matemáticas, y cuáles eran sus aplicaciones sociales.

En la actualidad los problemas descriptivos emergen con renovado interés con las propuestas curriculares que consideran que la resolución de problemas es una competencia básica en el desarrollo del pensamiento aritmético y algebraico. En este contexto adquieren particular importancia como testimonio del desarrollo histórico de las ideas matemáticas y de sus relaciones con el mundo real, y como ilustración de la continuidad de las matemáticas.

Porque hace miles de años la gente estaba intentado resolver problemas similares a los que resolvemos en la actualidad. Una revelación que a menudo resulta sorprendente para los estudiantes, pero que también resulta tranquilizadora, en el sentido de que se dan cuenta de que forman parte de un proceso que aún hoy continúa. (Swetz, 2014, p. 45)

\section{METODOLOGÍA PARA EL ANÁLISIS DE LOS PROBLEMAS DESCRIPTIVOS}

Cuando se quiere indagar acerca de cómo se ha configurado un contenido de las matemáticas de enseñanza, estudiando su evolución a lo largo de los diferentes momentos clave de la historia, hay que acudir al análisis de los libros de texto. Solo los libros de texto pueden aportar información sobre su planificación y puesta en práctica, al ser las únicas fuentes y registros documentales disponibles.

Para el estudio de los libros de texto, es útil servirse de la metodología denominada análisis didáctico. El análisis didáctico es un marco metodológico para la investigación en Educación Matemática, que tiene rasgos particulares y admite diversos enfoques funcionales y componentes, uno de ellos es el análisis de contenido (para más detalle ver Rico, Lupiáñez y Molina, 2013). 
Cuando el análisis de contenido es histórico-epistemológico, como se hace en Picado y Rico (2011) o Maz y Rico (2007), en tanto análisis de la formación de los objetos matemáticos a lo largo de su historia, se necesita de una metodología de aproximación global. Esto debido a que la metodología tradicional de estudiar textos aisladamente, o comparar varios textos entre sí, es insuficiente en la medida que tiende a desconsiderar las raíces y fuentes de las concepciones vertidas en el texto, su contexto social y cultural, y las particularidades propias del sistema educativo (Schubring, 1987). Es en este sentido en el que adquiere importancia revisar una buena selección de libros de texto, representativos de las grandes etapas en que se puede dividir la historia de las ideas matemáticas objeto de estudio.

El análisis de contenido matemático reflejado en los libros de texto se sustenta en subunidades de análisis o categorías. En el caso de los problemas descriptivos una de estas categorías es la lectura analítica del enunciado.

\section{LA LECTURA ANALÍTICA}

En sentido estricto la lectura analítica se aplica al planteamiento algebraico del problema. Una visión simplista consiste en creer que para ello basta observar una rigurosa traducción del lenguaje ordinario al lenguaje del álgebra, pero esto no siempre es posible porque no siempre las condiciones pueden ser fácilmente simbolizadas al estar envueltas de tal manera que es difícil descubrir su relación y sucesión.

Por eso, y con carácter general, se entiende que la lectura analítica del enunciado de un problema es hacerse cargo de todas las condiciones que explícita o implícitamente contenga el enunciado, y desenvolver las relaciones que las cantidades desconocidas tengan entre sí y con las cantidades conocidas.

Aunque la lectura analítica es la pieza fundamental del método cartesiano que conduce a la ecuación, también puede ser aplicada a la resolución aritmética de estos problemas, porque en la medida que la lectura analítica de un problema es reducirlo a una lista de cantidades y de relaciones entre cantidades, de ella se puede obtener la ecuación o la fórmula. La ecuación, se obtiene como resultado de igualar dos expresiones algebraicas que representan la misma cantidad, obtenidas al describir la relación aritmética que unas cantidades representadas con expresiones algebraicas tienen con otras que ya han sido previamente representadas por una letra o una expresión algebraica (Puig, 2003). La fórmula se obtiene al expresar en términos generales la relación de la cantidad desconocida con las cantidades conocidas, marcando así la lista de operaciones que hay que efectuar con los datos para obtener el 
valor que se busca ${ }^{3}$. Las fórmulas, traducidas al lenguaje usual dan las reglas generales de resolución ${ }^{4}$.

Así pues, según que la lectura analítica del enunciado conduzca a la ecuación o a la fórmula la lectura será considerada aritmética o algebraica. Esta diferenciación es coherente con el principio fundamental que distingue un proceso aritmético de un proceso algebraico, esto es la operación o no de la incógnita; es decir el que el número desconocido que se busca como solución del problema esté o no involucrado en el curso de las operaciones que se requieren para su determinación. Así, por ejemplo, el problema de hallar un número, tal que la suma de su doble y su mitad es igual a 25, conduce a operar el número desconocido $x$, a partir de la ecuación $2 x+\frac{x}{2}=25$ por lo que es claramente algebraico; pero si el problema pidiera encontrar un número que sea igual a la suma del doble y de la mitad de 10, el proceso de solución sería claramente aritmético $^{5}$.

\section{FUENTES DOCUMENTALES}

Las fuentes de información más antiguas para el estudio histórico-epistemológico de los problemas descriptivos se remontan a las culturas matemáticas mesopotámica y egipcia, anteriores a Cristo, transmitidas por las tabletas cuneiformes de Babilonia (2000-1600 a. C.) y los papiros egipcios ${ }^{6}$. También hay colecciones de problemas descriptivos en la matemática china, la más importante es la de los 247 problemas del Jiuzhang Suanshu (Los nueve capítulos del arte matemático, 100 d. C.). El legado de la antigua Grecia de estos problemas se puede consultar en los 46 problemas aritméticos de la Antología griega, la cual probablemente fuera reunida por Metrodoro (500 d. C.). En las matemáticas hindúes destacan las colecciones de problemas del manuscrito Bakhshali (300 d. C.) y de los matemáticos posteriores tales como Brahmagupta (628, d. C.), Mahavira (850 d. C.) y Bhaskara II (1150 d. C.). Los textos básicos de la Europa medieval son las colecciones de matemáticas recreativas de Beda, De Arithmeticis Prepositionibus (641 d. C.), y de Alcuino, Propositiones ad acuendos juvenes (775 d. C.), ambas con los mismos problemas salvo tres de ellos por lo que se sospecha que una es copia de la otra, la segunda redactada para una escuela de jóvenes talentos fundada por Carlomagno; así como el Liber abaci (1202) de

\footnotetext{
${ }^{3}$ Para Lacroix, las fórmulas son "unas meras indicaciones de la serie de operaciones que se deben ejecutar con las cantidades conocidas para hallar las desconocidas, y por consiguiente vienen a ser unas reglas generales escritas en lenguaje simbólico" (Lacroix, 1846, p. 20).

${ }^{4}$ En los métodos reglados se enuncia la serie de pasos que hay que ejecutar para que, sin necesidad de tener en cuenta la razón ni la explicación de los mismos, se pueda obtener la solución a un problema 0 ejercicio de una manera mecánica, breve y fácil de memorizar.

${ }^{5}$ El carácter general de los procesos aritméticos requiere que la cantidad desconocida que se busca como solución de la cuestión no esté involucrada en el curso de las operaciones que se requieren para su determinación (Peacock, 1842, p. 205, 206).

${ }^{6}$ El de Moscú (1850 a. C.), el Rhind (1650 a. C.), el de Rollin (1350 a. C.) y el de Harris (1167 a. C.).
} 
Leonardo de Pisa (Fibonacci), a quién se le debe la introducción en occidente de los métodos de la matemática islámica desarrollados durante el califato abasí (750-1000).

En la Europa renacentista, desde la aparición de la imprenta se multiplican los textos que compilan problemas descriptivos, desde ese momento se sucede un sinfín de grandes obras de autor que tratan específicamente el tema en la totalidad de la obra o en un capítulo específico. Ejemplos pioneros de esto son las obras de Bachet (1612) (considerada la primera obra de matemáticas recreativas), Oughtred (1653), Ozanam (1778), Vinot (1860), Ball (1892), Lucas (1894) y, más recientemente, Swetz (2014).

\section{PROBLEMA DE INVESTIGACIÓN}

Dado que los enunciados, el planteamiento y los métodos de resolución de estos problemas aparecen dispersos en los libros de aritmética, sin un criterio claro que los organice, parece conveniente hacer un estudio conjunto de los mismos. Este estudio debería contribuir a dar claridad y generalidad metodológica para fundamentar propuestas de enseñanza alternativas a una enseñanza cuyo enfoque pedagógico consiste en presentarlos como subproducto de otros aprendizajes, pero raramente como objeto de estudio por sí mismos.

Al centrarse únicamente en la resolución de problemas al modo de "ejercicio y práctica" para consolidar o aplicar los conocimientos adquiridos previamente, el currículo se olvida de mostrar una atención especial a aquellos aspectos de la resolución de problemas que tienen que ver con la producción de conocimientos significativos para el que aprende (Puig y Cerdán, 1995).

Es con esta intención, con la de producir no solo transmitir conocimiento, con la que se aborda el trabajo que aquí se presenta sobre los problemas descriptivos que emanan del primitivo problema de "las 100 aves de corral".

El origen del problema de "las cien aves de corral" se sitúa en China, en torno al siglo $\mathrm{V}$, pero ha sido replicado en el resto de las culturas matemáticas antiguas, como se aprecia en los siguientes ejemplos.

Ejemplo 1. "Un gallo vale 5 monedas; una gallina 3 monedas; y tres pollitos 1 moneda. Con 100 monedas compramos 100 de ellos. ¿Cuántos gallos, gallinas y pollitos tenemos?" (China, s. V, cit. Shen, Crossley y Lun, 1999, p. 416)

\section{Ejemplo 2}

Los pichones se venden a razón de 5 por 3 panas, los pájaros sarasa a razón de 7 por 5 panas y los pavos reales a razón de 3 por 9 panas. A un hombre se le dijo que trajera a esos precios 100 pájaros por 100 panas para que fueran una diversión para el hijo del rey, y fue enviado a hacerlo. ¿Qué cantidad entrega por cada una de las variedades de pájaro que compra? (Mahavira, 850 d. C., cit. Swetz 2014, p.189) 
Ejemplo 3. "Cinco palomas se compran por tres drammas; siete gruyas, por cinco; nueve gansos, por siete; y tres pavos reales, por nueve: traiga un centenar de estas aves por cien drammas, para gratificar el príncipe" (Bhashara, 1150, pp. 234-235).

\section{Ejemplo 4}

Con 100 monedas se compran naranjas [de la variedad] Wenzhou, naranjas verdes y naranjas doradas, 100 en total. Si una naranja Wenzhou cuesta 7 monedas, una naranja verde 3 monedas y una naranja dorada cuesta 1 moneda, ¿cuántas naranjas de las tres clases se compraron? (Yang Hui, 1275, cit. Shen, et al., 1999, p. 416)

Ejemplo 5. "1 pato cuesta 4 monedas, 5 gorriones cuestan 1 moneda 1 y 1 gallo cuesta 1 moneda. Uno compra a 100 aves a 100 monedas. ¿Cuántas aves puede comprar?" (Al-kashi, s. XV, cit. Shen, et al., 1999, p. 420)

En un principio el problema implicaba aves de corral, de ahí su título (Swetz, 2014, p. 36), pero en las versiones más antiguas que se conocen de este problema también aparecen enunciados con otros agentes, como se ve en los ejemplos siguientes.

Ejemplo 6. "Un total de 100 fanegas se distribuyen entre 100 personas, de tal modo que cada hombre recibe 3 fanegas, cada mujer 2 fanegas y cada niño $1 / 2$ fanega. ¿Cuántos hombres, mujeres y niños hay?” (Europa medieval, 775, cit. Swetz, 2014, p. 55)

\section{Ejemplo 7}

Dijo un mercader: quiero comprar 100 cerdos por 100 denarios; sin embargo pagaría 10 denarios por un macho, 5 por una hembra y uno por dos lechones. Diga, aquel que sepa, ¿cuántos machos, cuántas hembras y cuántos lechones debe haber de forma que no sobre ni falte ninguno? (Alcuino, 804, p. 1145)

También hay variantes de este problema que en vez de referirse a 100 ítems y otros 100 ítems, toman otra cantidad.

\section{Ejemplo 8}

Cierto paterfamilias disponía de 20 sirvientes. Ordenó que les fueran repartidos 20 modios de maíz del siguiente modo: que los hombres recibieran tres modios, las mujeres dos y los niños medio modio. Diga, quién pueda, ¿cuántos hombres, cuántas mujeres y cuántos niños debe haber? (Alcuino, 804, p. 1154)

\section{Ejemplo 9}

Cierto obispo ordenó repartir 12 panes entre el clero. Previó así que cada sacerdote recibiera dos panes; un diácono medio y un lector la cuarta parte. Obrando así el número de clérigos y de panes resulta el mismo. Diga, quién 
quiera, ¿cuántos sacerdotes, diáconos y lectores debe haber? (Alcuino, 804, p. 1155)

\section{Ejemplo 10}

Un hombre compra carne de cerdo a tres denarios la libra, de ternera a dos denarios la libra y de cabra a medio denario la libra. ¿Cómo ha de hacer para comprar siete libras de carne por siete denarios en total? (Fibonacci, 1202, p. 249)

\section{Ejemplo 11}

Un hombre compra 30 aves que son perdices, palomas y gorriones, por 30 denarios. Compra una perdiz por 3 denarios, una paloma por 2 denarios, y 2 gorriones para 1 denario, o sea 1 gorrión por 1/2 denario. Se pide, cuántas aves compra de cada clase (Fibonacci, 1202, p. 257)

\section{Ejemplo 12}

Un hombre quiere hacer un convite y da a su comprador 36 sueldos para que le compre tres clases de aves, como mirlos, tres por un sueldo, gallinas a 2 sueldos la pieza y capones a 3 sueldos la pieza. Y quiere 36 entre todos y que le cuesten 36 sueldos. Os pido ¿cuántos comprará de cada clase? (Ventallol, $1521 / 1621$, p. 472)

Ejemplo 13. "Uno fue a la plaza y halló tres suertes de aves, conviene a saber pájaros a blanca, zorzales a 3 blancas, charlas a 5 blancas, y compró 24 aves por 24 maravedís; pídese: ¿cuántas aves compró de cada suerte?” (Pérez de Moya, 1562/1998, p. 210)

\section{LA SOLUCIÓN DE FIBONACCI (1202)}

En el último apartado del capítulo 12 del Liber Abaci, denominado de las "reglas para mezclar cosas análogas", Fibonacci (1202, p. 257) resuelve este tipo de problemas y dice explícitamente que lo hace "por el método de las aleaciones"?.

Un hombre compra 30 aves que son perdices, palomas y gorriones, por 30 denarios. Compra una perdiz por 3 denarios, una paloma por 2 denarios, y 2 gorriones para 1 denario, o sea 1 gorrión por $\frac{1}{2}$ denario. Se pide, cuántas aves compra de cada clase (figura 2).

\footnotetext{
${ }^{7}$ Recordemos que la aleación es uno de los contextos en que se desenvuelven los problemas de aligación.
} 


\begin{tabular}{|c|c|c|}
\hline perdices & gorriones & Divide los 30 denarios por las 30 aves; el cociente será 1 denario. \\
\hline 1 & 4 & $\begin{array}{l}\text { Di, por consiguiente, tengo dinero con } 1 / 2 \text {, dinero con } 2 \text {, y dinero } \\
\text { con } 3 \text {, y deseo tener dinero con } 1 \text {. Es claro que como en los }\end{array}$ \\
\hline \multicolumn{2}{|c|}{ Primera mezcla } & $\begin{array}{l}\text { problemas similares el procedimiento es por el método de } \\
\text { aleaciones, ya que tenemos un número entero de aves. Por }\end{array}$ \\
\hline 1 & 4 & consiguiente, dado que la clase más barata de pájaros es igual en \\
\hline 6 & 1 & número que la clase más cara, di, tengo dinero con $1 / 2$, y dinero \\
\hline \multicolumn{2}{|c|}{2} & $\begin{array}{l}\text { con } 2 \text {, y dinero con } 3 \text {, y deseo tener dinero con } 1 \text {; o sea que, tengo } \\
\text { dinero con } 1 \text {, y dinero con } 4 \text {, y dinero con } 6 \text {, y deseo tener dinero } \\
\text { con } 2 \text {; haz con los gorriones y las perdices la primera aleación, y } \\
\text { saldrá } 5 \text { aves por } 5 \text { denarios, o } 4 \text { gorriones y } 1 \text { perdiz, y haz una }\end{array}$ \\
\hline 1 & 2 & $\begin{array}{l}\text { segunda aleación con los gorriones y las palomas, y tendrás } 3 \\
\text { aves para } 3 \text { denarios, o } 2 \text { gorriones y } 1 \text { paloma; después a fin de }\end{array}$ \\
\hline \multicolumn{2}{|c|}{ Segunda mezcla } & $\begin{array}{l}\text { tener } 30 \text { aves en la aleación, pon la primera aleación tres veces, } \\
\text { que serán } 12 \text { gorriones y } 3 \text { perdices, y quedarán } 15 \text { aves para }\end{array}$ \\
\hline 1 & 2 & alear, para cuál pon la segunda aleación cinco veces, y tendrás 10 \\
\hline \multicolumn{2}{|c|}{2} & $\begin{array}{l}\text { gorriones y } 5 \text { palomas, y así de las anteriormente dichas } 30 \text { aves } \\
\text { habrá } 22 \text { gorriones y } 5 \text { palomas y } 3 \text { perdices, como se ha } \\
\text { mostrado en el problema. (Fibonacci, 1202, p. 256) }\end{array}$ \\
\hline
\end{tabular}

Figura 2. Solución por el método de las aleaciones

Para Fibonacci este problema es análogo a los de "aleación alternada total", y lo resuelve como tal, aplicando la regla de la cual ya ha dado cuenta en un apartado precedente del capítulo 12 .

\section{LA REGLA DE ALIGACIÓN}

Bajo el nombre de regla de aligación se conoce el procedimiento para resolver los problemas relacionados con la mezcla de sustancias de distinta calidad y precio. $\mathrm{Si}$ son metales se llama aleación y si líquidos se llama disolución.

La regla de aligación tiene por objeto resolver dos tipos de cuestiones: (1) hallar el precio de la mezcla de varias sustancias, conociendo el número de unidades que se mezclan y su precio; y (2) hallar el número de unidades de precio conocido que han de mezclarse, para que la mezcla tenga un precio determinado. A los problemas del primer tipo de se les ha denominado tradicionalmente "aleación medial" y a los del segundo tipo "alternada". En los libros de texto modernos (por ejemplo, en Vallín, 1862, p. 174) se les llama aligación directa e inversa respectivamente. En el contexto de las aleaciones, estos dos tipos de problemas se particularizan en hallar la ley de aleación de un metal noble (cantidad de metal noble por unidad de peso) y hallar las cantidades que hay que fundir de cada metal. Ejemplos genéricos de ambos tipos son los siguientes.

Medial. Se mezclan $c \mathrm{~kg}$ de café a $\mathrm{p}$ ptas. $\mathrm{Kg}$ con $c^{\prime} \mathrm{kg}$ a $p^{\prime}$ ptas. y con $c^{\prime \prime} \mathrm{kg}$ a $p$ "' ptas. ¿A cómo resulta el precio de la mezcla? (García, 1959, p. 108) 
Alternada. Se quiere formar una mezcla de $T \mathrm{~kg}$ de café a $P$ ptas. el $\mathrm{kg}$ con dos clases distintas. El precio de la primera es $p$ ptas. el $\mathrm{kg}$ y el de la segunda $p$ ' ptas. el $\mathrm{kg}$. ¿Cuántos kg habrá de cada clase? (García, 1959, p. 109)

La resolución de esto dos tipos de problema se sustenta en la condición fundamental de toda mezcla, que se puede enunciar de dos maneras equivalentes: una es que después de hecha la mezcla debe resultar el mismo precio que por separado (I), y la otra es que lo que se gana con el producto de menor valor ha de compensar lo que se pierde con el de mayor valor (II).

Llamando A y B a las cantidades mezcladas, $\mathrm{p}$ y $\mathrm{q}$ a sus precios respectivos y $\mathrm{P}_{\mathrm{m}}$ al precio medio o por unidad de mezcla, estos dos enunciados dan lugar a las siguientes relaciones entre cantidades.

I. $A p+B q=(A+B) P_{m}$ (después de hecha la mezcla debe resultar el mismo precio que por separado)

II. $A\left(p-P_{m}\right)=B\left(P_{m}-q\right)$ (lo que se gana con el producto de menor valor ha de compensar lo que se pierde con el de mayor valor)

Obsérvese que ambas relaciones son equivalente en el sentido de que cualquiera de ellas se deduce de la otra por transformaciones algebraicas.

$$
A p+B q=(A+B) P_{m} \Leftrightarrow A\left(p-P_{m}\right)=B\left(P_{m}-q\right)
$$

De estas relaciones se obtienen las fórmulas generales que resuelven los dos tipos de problemas de aligación. De la relación I se sigue la fórmula que da el precio medio o por unidad de mezcla.

$$
A p+B q=(A+B) P_{m} \rightarrow P_{m}=\frac{A p+B q}{A+B}(\mathrm{~F} . \mathrm{I})
$$

De esta fórmula se desprende la siguiente regla.

Para sacar este precio medio se multiplica cada género por el precio a que se pagó, se suman unos con otros los productos; claro está que su suma será el valor de toda la mezcla. Por consiguiente con partir este valor por la suma de todos los géneros, el cociente será el precio medio o común de todos. (Bails, 1790, p. 133)

De la relación II se sigue la fórmula que da la relación entre las cantidades que se han de mezclar.

$$
A\left(p-P_{m}\right)=B\left(P_{m}-q\right) \rightarrow \frac{A}{B}=\frac{P_{m}-q}{p-P_{m}}(\mathrm{~F} . \mathrm{II})
$$

En este caso, no hay una solución, ya que la fórmula solo da la relación en que deben entrar las cantidades.

Cuando la mezcla es de más de dos componentes el planteamiento del problema consiste en reducirlo al caso que ya se sabe resolver, que es el de dos componentes. 
Así, por ejemplo, si fueran tres las componentes, habría dos cuyo precio sería mayor o menor que el medio, suponiendo que el precio de dos componentes fuera menor que el medio la relación entre las cantidades sería:

III. $A\left(p-P_{m}\right)+B\left(q-P_{m}\right)+B\left(q-P_{m}\right)=C\left(P_{m}-r\right)$

En este caso, como la solución es indeterminada, y hay infinitas soluciones, una posible solución es la que resulta al tomar la misma cantidad de las componentes de precio menor, es decir $A=B$, entonces:

$$
A\left(p-P_{m}\right)+A\left(q-P_{m}\right)=C\left(P_{m}-r\right) \rightarrow \frac{A}{C}=\frac{B}{C}=\frac{P_{m}-r}{p-P_{m}+q-P_{m}}(\mathrm{~F} . \mathrm{III})
$$

\section{LA DISPOSICIÓN PRÁCTICA DE LOS DATOS}

En la práctica, la disposición de los datos consiste en ordenar los precios de las sustancias, de mayor a menor (o de menor a mayor), arriba y abajo (figura 3, izquierda.) o a la izquierda y derecha (figura 3, derecha.), del precio medio que está a la izquierda en otra columna o abajo en otra fila. Se ponen las diferencias invirtiendo (alternando) su posición, a la derecha o encima, porque la razón entre las diferencias es la razón inversa que se busca.

\begin{tabular}{|lccl|}
\hline Disposición en vertical & \multicolumn{2}{c|}{ Disposición en horizontal } \\
\hline$p$ & $P_{m}-q$ & $P_{m}$ el precio medio & Diferencias $P_{m}-q p-P_{m}$ \\
$P_{m}$ & & $p$ el precio mayor & Diferencias $P_{m}-q p-P_{m}$ \\
$q$ & $p-P_{m}$ & $q$ el precio menor & \\
Precio & $\begin{array}{l}\text { Diferencias } \\
\text { altemadas }\end{array}$ & & Preciomedio $P_{M}$ \\
\hline
\end{tabular}

Figura 3. Disposición práctica de la regla de aligación para dos componentes Cuando al lado de un precio hay varias diferencias, se suman (ver figura 4). 


\begin{tabular}{|cc|}
$P_{a}$ & $\left(P_{m}-P_{b}\right)+\left(P_{m}-P_{c}\right)$ \\
$P_{m}$ & \\
$P_{b}$ & $P_{a}-P_{m}$ \\
$P_{c}$ & $P_{a}-P_{m}$ \\
\hline
\end{tabular}

Figura 4. Disposición práctica de la regla de aligación para tres componentes El ejemplo que se muestra en la figura 5 ilustra la regla práctica para el caso de tres componentes.

Supongamos que hay tres clases de té: uno de a 80 reales la libra, otro de a 60 reales y otro de a 57, y que se pregunte en qué razón se han de mezclar para que se pueda vender a 72 rs. la libra

Dispondremos los términos en esta forma (A) y hallo que por cada 8 libras de a 60 y de a 57 que mezcle, deberé mezclar 27 del de a 80 rs.

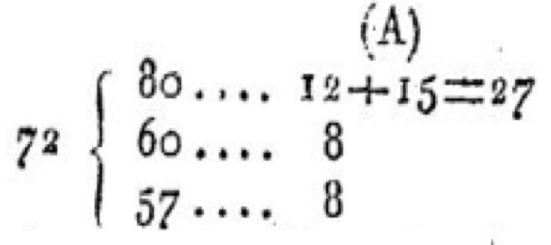

Figura 5. Regla práctica para tres componentes (Vallejo, 1841, p. 365)

En las aritméticas comerciales, se le daba mucha importancia a esta regla y a su disposición práctica, y esta es la que utiliza Fibonacci para resolver el problema de las 30 aves. Pero antes de explicar esto es necesario considerar los dos casos que se pueden dar en la aligación alternada.

\section{ALIGACIÓN ALTERNADA O INVERSA TOTAL Y PARCIAL}

Para que la solución de los problemas de aligación alternada no sea indeterminada, se suele añadir un dato extra, ya sea la cantidad total de mezcla que se desea obtener, entonces los problemas se llaman de Aligación Total; o ya sea la cantidad con que entra una de las componentes de la mezcla, entonces, los problemas se llaman de Aligación Parcial.

Para resolver estos dos casos es necesario combinar la regla de aligación alternada con el prorrateo (repartir proporcionalmente) de la cantidad total o parcial conocida en la razón en que entran las cantidades en la mezcla.

Los ejemplos que se muestran en la figura 6 ilustran estos dos casos (Dalmau, 1943, p. 270). 


\section{LA SOLUCIÓN DE FIBONACCI A LA LUZ DE LA REGLA DE ALIGACIÓN TOTAL}

Los precios son: perdices a 3 , palomas a 2 , gorriones a $\frac{1}{2}$; y el precio medio es 1 ya que viene de 30 aves a 30 denarios, y la cantidad total es 30 aves. Para averiguar cuántas aves compra de cada clase bajo esas condiciones, Fibonacci plantea la regla de aligación alternada total, en el caso de tres componentes dos de las cuales tienen el precio mayor que el precio medio.

Total. ¿Cuántos quintales $m$. de harina de a 20 ptas. y de a 16 ptas. deberán tomarse para obtener 60 qq. m. de 17,75 ptas.?

$1^{2}$ clase $\mathrm{x}$ quint. m. de 20 ptas.

$17 \cdot 75$

$2^{\mathbf{2}} \quad$ u $\mathrm{z} u$ u 16 "

$2^{\prime} 25$

Suma de especies: $x+y=60$ qint. $m$. Suma de diferencias: $175+2 ' 25=4$.

Dividiendo 60 quint. $m$. en partes proporcionales a las diferencias obtenidas tendremos

$$
\begin{array}{ccc}
\text { Para la } 1^{2} \text { clase: } & 4: 60:: 1^{\prime} 75: \mathrm{x} . & \mathrm{x}=26^{\prime} 25 \mathrm{qq} . \\
\text { Para 1a } 2^{2} \text { : }: & 4: 60:: 2^{\prime} 25: \mathrm{x} . & \mathrm{x}=33^{\prime} 75 \mathrm{qq} . \\
\text { Cantidad de mezcla que se ha dado } & 60^{\prime} 00
\end{array}
$$

Parcial. Un comerciante en cereales tiene maiz de a 14 ptas. el $\mathrm{HL}$, y $20 \mathrm{HL}$. de a 11. ¿Cuäntos $\mathrm{Hl}$. de la primera clase deberá mezclar con los 20 de la segunda, a fon de vender el Hl. de mezcla a 13 ptas.?

$x$ Hl. de 14 ptas.

13

$20 " 11 *$

$20: x:: 1: 2 . \quad x=40 \mathrm{HL}$.

Figura 6. Combinación de la regla de aligación alternada con el prorrateo Ante todo, para evitar el precio fraccionario que vale 1 gorrión, $\frac{1}{2}$ sueldo, dobla las cantidades de modo que, en vez de 3,2 y $\frac{1}{2}$, aves a precio medio 1 , tiene 6,4 y 1 aves a precio medio 2. Con estos datos plantea una regla de aligación con dos componentes, los gorriones y las perdices, usando la disposición práctica horizontal, y halla que de 5 aves 4 han de ser gorriones y 1 perdiz. A continuación plantea una segunda aligación con los gorriones y las palomas, y halla que de 3 aves 2 han de ser gorriones y 1 paloma (tabla 1 ).

A fin de tener 30 aves en total, como no puede prorratear a 5 y 3, como cabría esperar, porque obtendría cantidades no enteras, Fibonacci toma los múltiplos de 5 y 
de 3 que juntos entren en 30, esto son 3 veces las 5 aves de la primera combinación y 5 veces las 3 aves de la segunda, lo que hace un total de 12 gorriones y 3 perdices más 10 gorriones y 5 palomas, que son 22 gorriones, 5 palomas y 3 perdices.

Tabla 1

Disposición práctica horizontal de Fibonacci

\begin{tabular}{ccc}
\hline Perdices & \multicolumn{2}{c}{ Gorriones } \\
\hline \multicolumn{3}{c}{ Primera aligación } \\
1 & 4 \\
6 & 2 & 1 \\
& & \\
1 & Segunda aligación \\
4 & & 2 \\
& 2 & 1 \\
\hline
\end{tabular}

\section{LA SOLUCIÓN DE VENTALLOL}

Tres siglos después, Ventallol (1521/1621), resuelve un problema similar de "aves" pero su argumentación es diferente.

Un hombre quiere hacer un convite y dio a su comprador 36 sueldos para que le compre tres suertes de volatería [clases de aves], es a saber, mierlas, 3 por un sueldo, gallinas, a 2 sueldos la pieza y capones, a 3 sueldos la pieza; $y$ quiere, que entre todos sean 36, y que le cuesten 36 sueldos. Pregunto, ¿cuántos comprará de cada suerte?

Compra los 36 de aquellos que valen menos, esto es, mierlas, y hallarás que cuestan 12 sueldos. Sácalos de 36, quedan 24. Veas primero qué vale una mierla y hallarás que vale 1/3 de sueldo. Ahora mira cuánto vale más una gallina que una mierla y hallarás $12 / 3$, ponlos aparte. Después mira cuánto vale más un capón que una mierla, y hallarás 2 sueldos y 2/3. Ahora haz tercios de todo, es a saber, de $12 / 3$ y serán 5/3, y así mismo de 2 2/3 y serán $8 / 3$, después harás de 24 sueldos tercios, y serán 72/3. Ahora de estos 72 haz tales dos partes que la una se pueda partir por 5 y la otra por 8 y que no sobre nada en la partición. Esto lo hallarás así: saca tantas veces 5 de 72, hasta que quede un número que se pueda partir por 8 justamente, y hallarás que será 32; y el otro será 40. Parte 32 por 8 y vendrán 4; después parte 40 por 5, y vendrán 8 . Y así ves que habrá 4 capones y 8 gallinas, que son 12, hasta 36 van 24, y tantas mierlas habrá. Y asi harás de todas las semejantes. (Ventallol, 1521/1621, pp. 472-473) 
Ante todo Ventallol averigua el coste de comprar las 36 aves como si todas valieran lo que las mierlas, que es la que vale menos. A ese precio gastaría 12 sueldos y le quedarían por gastar 24 sueldos. Esto 24 sueldos los puede usar para pagar el plus o diferencia que tienen las otras aves respecto a las mierlas, por eso calcula esas diferencias, que son $5 / 3$ y $8 / 3$, y cuántas de esas diferencias entran en los 24 sueldos. O lo que es lo mismo, cuántas veces $5 / 3$ y $8 / 3$ juntos hacen $24=72 / 3$ sueldos, $\mathrm{y}$ eso serán los conejos y las gallinas. Restando de 36 la suma de los conejos y las perdices se tiene las mierlas.

\section{LA SOLUCIÓN DEL PROBLEMA A LA LUZ DEL MÉTODO DE POSICIÓN REMOCIÓN}

El método que utiliza Ventallol, era conocido en las aritméticas provenzales bajo el nombre de "posición-remoción". Parece ser que este método, que se presentaba junto con los de "falsa posición", no tiene equivalente en los tratados de ábaco italianos (Van Egmon, 1988, cit. Malet, 1998).

Pérez de Moya, recoge el método en su Arithmetica práctica (1562/1998):

Uno compró 100 piezas, entre perdices y conejos, por 94 reales. Demando, valiendo cada perdiz 32 maravedís y medio, y un conejo 30, ¿cuántos conejos compró?

Ésta y sus semejantes se hacen proponiendo que las 100 piezas eran todas conejos, que en este ejemplo es lo que vale menos, los cuales valiendo cada uno 30 maravedís montarán 3000. Estos 3000 redúcelos a reales y serán 88 y $4 / 17$ abos de real, réstalos de 94 reales que se gastaron en todo y restarán 5 reales y 13/17 abos. Mira ahora la diferencia que hay del precio de un conejo al de la perdiz y hallarás ser dos maravedís y medio, por los cuales partirás los maravedís que valen los 5 reales y 13/17 abos de real, y vendrá al cociente 78 y 2 quintos; $y$ tantas fueron las perdices, y lo que falta hasta 100, que son 21 y 3 quintos, fueron los conejos. (Pérez de Moya, 1562/1998, p. $210)^{8}$

Como se ha explicado antes, el método consiste en averiguar el coste de comprar todas las piezas al precio de la que vale menos, en este caso los conejos. Esto deja $5+\frac{13}{17}$ reales para el plus de más que cuestan las predices con respecto a los conejos.

Para ello basta con ver cuántas veces cabe ese plus de $2+\frac{1}{2}$ maravedís en $5+\frac{13}{17}$ reales, y eso serán las perdices.

Un texto de 1275 d. C. cuyo autor es Yang Hui ofrece un rastro chino del método de posición - remoción. El enunciado es el siguiente.

\footnotetext{
${ }^{8}$ Nota: 1 real $=34$ maravedís
} 
Con 100 monedas se compran naranjas [de la variedad] Wenzhou, naranjas verdes y naranjas doradas, 100 en total. Si una naranja Wenzhou cuesta 7 monedas, una naranja verde 3 monedas y una naranja dorada cuesta 1 moneda, ¿cuántas naranjas de las tres clases se compraron?

De 3 veces 100 monedas resta 100 monedas; de 3 veces el precio de las naranjas Wenzhou, i.e. 21, resta 1; el resto es 20. De 3 veces el precio de las naranjas verdes, i.e. 9, resta 1; el resto 8. La suma de los restos es 28. Divide 200 por 28, tendrás el entero $6\left[i^{7+4 / 28}\right.$ ?]. Este es el número que se busca; 6 naranjas Wenzhou y 6 naranjas verdes respectivamente. Después $(200-6 \times 28) \div 8=4$, y esta es la diferencia entre las cantidades de naranjas Wenzhou y verdes. Por tanto, su suma es 16, mientras que el número de las naranjas doradas que se busca es 84. (Yang, 1275, cit. Shen, et al., 1999, p. 416)

Como se ha visto antes, Yang evita el precio fraccionario, multiplicando por 3 todos los precios. Esto le da 300 monedas, para comprar naranjas de a 21, 9 y 1 moneda. A continuación aparta de las 300 monedas, lo que costaría comprar 100 de las naranjas de menor valor, que es 100. La diferencia, 200, es lo que queda para pagar el plus de lo que valen las otras naranjas respecto a la de menor valor, o sea $21-1=20 \mathrm{y}$ $9-1=8$. Ahora solo queda hallar de cuántas veces 20 y 8 está formado 200 . Aunque es un misterio como lo averigua, tal vez por ensayo y error, obtiene como resultado 6 y 10 respectivamente, lo que es correcto.

\section{OTRA SOLUCIÓN EN LA MATEMÁTICA TRADICIONAL CHINA}

En la matemática china, aparece el problema de las 100 aves en un texto del siglo V. "Un gallo vale 5 monedas, una gallina 3 monedas, y 3 pollitos 1 moneda. Con 100 monedas compramos 100 de ellos. ¿Cuántos gallos, gallinas y pollos hay?" (Zhang, Qiujian's Manual de Matemáticas, S. v., cit. Shen, et al. 1999, p. 416).

Una explicación del método de resolución que se encuentra en los textos chinos (siglos más tarde) es la siguiente (tabla 2).

Por 1 gallina y 3 pollos, i.e. por 4 aves de corral, el coste es de 4 monedas, y asi para 100 aves de corral, 75 son pollos y 25 son gallinas. Si aumentamos los 75 pollos en 3, y disminuimos las gallinas en 3, el número de aves de corral permanece inalterado, mientras que el número de monedas disminuye en 8. Si después de esto, se cambian 4 gallinas por 4 gallos, entonces el número de monedas también permanece inalterado [las $4 \times 5$ monedas de los gallos compensan los $3 \times 4$ monedas de las gallinas junto con las 8 monedas de antes], i.e. 100. Esto significa que cada vez que aumentamos los 75 pollos en 3 , y disminuimos las 25 gallinas en 7, mientras aumentamos los gallos en 4, los números de aves de corral y de monedas permanecen inalterados. Como 
hay 25 gallinas, a lo sumo se puede hacer esto $25 / 7=3$ veces, lo que da tres conjuntos de respuestas. (Ding Quzhhong, cit., Shen, et al., 1999, p. 418)

Tabla 2

Explicación al método de resolución chino

\begin{tabular}{ccc}
\hline Gallinas & Gallos & Pollos \\
\hline $25-7=18$ & 4 & $75+3=78$ \\
$25-14=11$ & 18 & $75+6=81$ \\
$25-21=4$ & 12 & $75+9=84$ \\
\hline
\end{tabular}

\section{LA LECTURA CARTESIANA}

Al asignar las letras a las cantidades que se buscan, las condiciones del problema dan lugar a un sistema de dos ecuaciones lineales. El siguiente ejemplo muestra el proceso de resolución tal y como se plantea en un texto del siglo XVIII.

Cien personas gastaron 100 doblones, cada varón 3, cada mujer 1, cada niño medio y cada criado un séptimo. Pídese, en particular, el número de los hombres, de las mujeres, niños y criados.

Supongo, como antes, que el número de los varones sea $v$, el de las mujeres $m$, el de los niños $n$, y el de los criados c; y porque las suma de todos es 100, será la primera igualación [ecuación], $v+m+n+c \Omega 100$ [usa $\Omega$ en vez de $=$ ]. Y porque cada varón gastó 3 doblones; cada mujer 1, cada niño medio, y cada criado un séptimo; y todos juntos 100, se formara de estas la segunda igualación, que es $3 v+1 m+\frac{n}{2}+\frac{c}{7} \Omega 100$, y estas dos igualaciones formarán la columna de dirección. Despejando ahora por antitesis la primera, será $v \Omega 100-m-n-c$, que pongo en la a columna del retorno; y sustituyendo este valor de $v$ en la segunda igualación de la columna de dirección, y hecha la transposición, resulta $2 m+3 n-\frac{n}{2}+3 c-\frac{c}{7} \Omega 20, y$ quitando los quebrados, es $28 m+35 n+40 c \Omega 2800, y$ partiendo todo por 28 , es $m+\frac{35 n}{28}+\frac{40 c}{28} \Omega 100, y$ despejando la incógnita $m$, es $m \Omega 100-\frac{35 n}{28}-\frac{40 c}{28}$, que escribo en la columna de retorno, como aquí se sigue 


$$
\left.\begin{array}{l}
v+m+n+c \Omega 100 \\
3 v+1 m+\frac{n}{2}+\frac{c}{7} \Omega 100
\end{array}\right] \begin{aligned}
& m \Omega 100-\frac{35 n}{28}-\frac{40 c}{28} \\
& v \Omega 100-m-n-c
\end{aligned}
$$

Donde se ve ser la cuestión indeterminada por no poderse excluir la n, ni la c, conque en lugar de dichas letras se podrán sustituir cualesquiera números, mientras que sean tales que el valor que dieren a los quebrados se pueda restar de 100.

Pero para dar todas las respuestas que en enteros son posibles, se obrará como en la cuestión pasada, sustituyendo primeramente en lugar de c el número 7 que es el primero que multiplicado por 40 se puede partir justamente y sin resto por 28, y continuando las sustituciones de los múltiplos de 7, por su orden, se hallarán las nueve igualaciones siguientes.

$\begin{array}{lll}c \Omega 7 & \text { da } & m \Omega 90-\frac{35 n}{28} \\ c \Omega 14 & \text { da } & m \Omega 80-\frac{35 n}{28} \\ c \Omega 21 & \text { da } & m \Omega 70-\frac{35 n}{28} \\ c \Omega 28 & \text { da } & m \Omega 60-\frac{35 n}{28} \\ c \Omega 35 & \text { da } & m \Omega 50-\frac{35 n}{28} \\ c \Omega 42 & \text { da } & m \Omega 40-\frac{35 n}{28} \\ c \Omega 49 & \text { da } & m \Omega 30-\frac{35 n}{28} \\ c \Omega 56 & \text { da } & m \Omega 20-\frac{35 n}{28} \\ c \Omega 63 & \text { da } & m \Omega 10-\frac{35 n}{28}\end{array}$

Para acabar de conocer el valor de $m$ se harán las sustituciones de los enteros en lugar de $n$ en todas las sobredichas igualaciones, y empezando por la primera se sustituirá primeramente el número 4, que es el menor que multiplicado por 35 puede partirse justamente por 28, luego se continuará, sustituyendo los demás múltiplos del 4 en la misma igualación, hasta que el valor del quebrado no se pueda restar de 90, y se tendrán todas las 
respuestas que puedan salir de dicha igualación. Esto mismo se hará en cada una de las demás, y se tendrán las 81 resoluciones siguientes, (...) (Tosca, 1757, pp. 168-170).

Como se ve, como hay más incógnitas que ecuaciones la solución no es única. Para que el sistema sea determinado se precisa entonces alguna condición complementaria, por ejemplo que $x, y, z$ sean enteros. Esto es lo que ocurre, por ejemplo, en el problema siguiente.

Un hombre quiere hacer un convite y dio a su comprador 36 sueldos para que le compre tres suertes de volatería [aves], es a saber, mierlas, 3 por un sueldo, gallinas, a 2 sueldos la pieza y capones, a 3 sueldos la pieza; $y$ quiere, que entre todos sean 36, y que le cuesten 36 sueldos. Pregunto, ¿cuántos comprará de cada suerte?

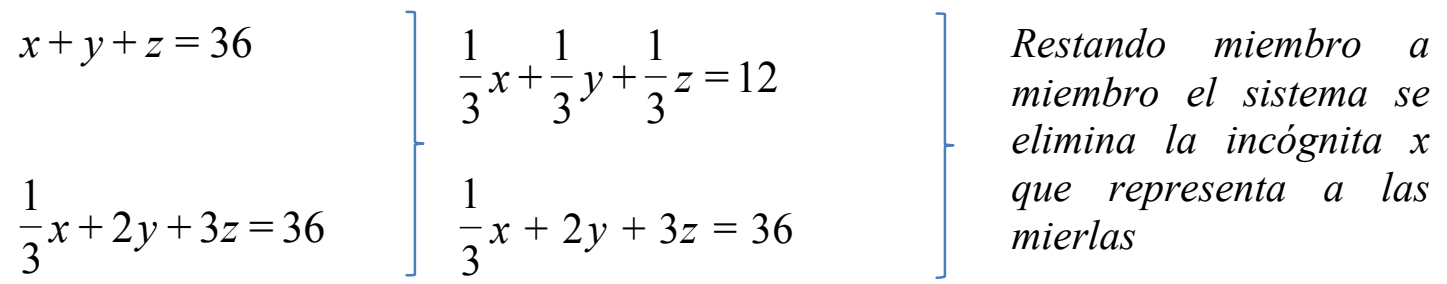

$$
1+\frac{2}{3} y+2+\frac{2}{3} \quad z=24 \rightarrow \frac{5}{3} y+\frac{8}{3} z=\frac{72}{3} \rightarrow 5 y+8 z=72
$$

El problema queda resuelto descomponiendo 72 en dos partes tales que una sea múltiplo de 8 y la otra múltiplo de 5. Obsérvese la analogía entre las operaciones para resolver el sistema por el método de igualación y las que se efectúan con el método de "posición-remoción".

Lo mismo ocurre en el problema de Fibonacci, donde la condición complementaria es que $x, y, z$ son enteros $<30$.

Un hombre compra 30 aves que son perdices, palomas y gorriones, por 30 denarios. Compra una perdiz por 3 denarios, una paloma por 2 denarios, y 2 gorriones para 1 denario, o sea 1 gorrión por 1/2 denario. Se pide, cuántas aves compra de cada clase. (Fibonacci., 1202, p. 257)

$$
\begin{aligned}
& \left.\begin{array}{l}
x+y+z=30 \\
3 x+2 y+z / 2=30
\end{array}\right\} x+y+z=30 \\
& 3 x+2 y+\frac{(30-x-y)}{2}=30 \rightarrow 5 x+3 y=30,
\end{aligned}
$$

Esta es una ecuación indeterminada que para $x, y, z$ enteros $<30$; da $x=3, y=5, z=$ 22. 


\section{EPÍlOGO}

Aunque los problemas descriptivos como el de las "100 aves de corral" tienen cada vez menos presencia en la enseñanza actual, el caso es que estos problemas forman parte del legado matemática que nos ha transmitido la historia.

En este artículo se ha pretendido dar cuenta de una parte de este legado, y de cómo ha evolucionado desde las culturas matemáticas antiguas hasta nuestros días. Se han mostrado sus distintas lecturas analíticas, aritméticas y cartesianas, y se han analizado y resaltado sus métodos de resolución: aligación, posición-remoción y ecuaciones.

Se aporta así un conocimiento matemático cuya riqueza forma parte del pensamiento aritmético y algebraico que ha desarrollado la humanidad, o, parafraseando a Freudenthal (1981) de los procesos cognitivos que ha desarrollado la humanidad para ser competente en esta materia.

Toda esta información, puede ser útil para la enseñanza, porque aporta claridad y organización metodológica, ya que los problemas adquieren protagonismo como objeto de estudio organizado, y no como subproducto de otros aprendizajes, al mostrar y analizar sus distintos métodos de resolución se pone el énfasis en la producción más que en la transmisión de conocimiento.

Además, también puede ser útil para el investigador en pensamiento numérico y algebraico porque aporta un modelo de análisis histórico-epistemológico para el estudio de los problemas clásicos, no como piezas sueltas de un contenido matemático sino en relación con sus raíces y evolución.

Ahora el reto de los profesores e investigadores es mantener viva esa riqueza de conocimientos, evitar que caiga en el olvido, y adaptarlo a la enseñanza y a las características de los estudiantes, atendiendo al objetivo curricular que considera la resolución de problemas como una competencia básica.

\section{Agradecimiento}

Este trabajo se ha realizado en el marco de los proyectos de investigación del Ministerio de Ciencia e Innovación: EDU2012-35638 y EDU2011-27168.

\section{REFERENCIAS}

Alcuino (1863). Propositiones ad acuendos juvenes. En J. P. Migne (Ed.), Patrologiae cursus completus: patrologiae latinae (Tomo 101, columnas 1143-1160; original, 775). París, Francia.

Bails, B. (1790). Arismética para negociantes. Madrid, España: La viuda de Ibarra. Bhaskara, A. (1150). Lilavati (H. T. Colebrooke, Trad.; original, 1817). Londres, Reino Unido: Murray.

Corachán, J. B. (1699). Arithmetica demonstrada theorico-practica para lo mathematico y mercantil. Valencia, España: Jaime de Bordazar. 
Dalmáu, J. (1943). Soluciones analíticas. Libro del maestro. Gerona, España: Dalmáu Carles.

Fibonacci, L. (2002). Liber Abacci. En L. Sigler (Ed.), Fibonacci's Liber Abaci a Translation into Modern English of Leonardo Pisano's Book of Calculation (Original, 1202). Nueva York, NY: Springer.

Freudhental, H. (1981). Major problems of mathematics education. Educational Studies in Mathematics, 12(2) 133-150.

García, J. (1959). Matemáticas tercer curso. Alcoy, España: Editorial Marfil.

Lacroix, S. F. (1846). Curso completo elemental de matemáticas puras (Josef Rebollo y Morales, trad.). Tomo II. Algebra ( $6^{\mathrm{a}}$ ed.). Madrid, España: Imprenta Nacional.

Malet, A. (1998). Summa de l'art d'Aritmètica. Francesc Santcliment. Introducció, transcripció i notes a cura d'Antoni Malet [Suma del arte de Aritmética. Francisco Santcliment. Introducción, transcripción y notas al cuidado de Antoni Malet]. Barcelona, España: Eumo.

Maz, A. y Rico, L. (2007). Situaciones asociadas a los números negativos en textos de matemáticas españoles de los siglos XVIII y XIX. $P N A, 1(3), 113-123$.

Meavilla, J. L. (2000). Historia de las matemáticas: métodos no algebraicos para la resolución de problemas. $S U M A, 34,81-85$.

Peacock, G. (1842). Treatise on Algebra-Vol.1 [Tratado del álgebra. Vol. 1]. Cambridge, Reino Unido: University Press.

Picado, M. y Rico, L. (2011). Análisis de contenido en textos históricos de matemáticas. PNA, 6(1), 11-27.

Pérez de Moya, J. (1998). Arithmetica práctica y especulativa (Original, 1562). Salamanca, España: Biblioteca Castro.

Puig, L. (2003). Historia de las ideas algebraicas: componentes y preguntas de investigación desde el punto de vista de la matemática educativa. En E. Castro, P. Flores, T. Ortega, L. Rico y A. Vallecillos (Eds.). Investigación en Educación Matemática. Actas del VII Simposio de la SEIEM (pp. 97-108). Granada, España: SEIEM y Universidad de Granada.

Puig, L. y Cerdán, F. (1995) Problemas aritméticos escolares. Madrid, España: Síntesis.

Rico, L., Lupiáñez, J. L. y Molina, M. (2013). Análisis didáctico en Educación Matemática. Metodología de investigación, formación de profesores e innovación curricular. Granada, España: Comares.

Santcliment, F. (1998). Summa de l'art d'aritmètica [Suma del arte de la artimética] (Original, 1482). En A. Malet (Ed.), Summa de l'art d'Aritmètica. Francesc Santcliment. Introducció, transcripció i notes a cura d'Antoni Malet. Barcelona, España: Eumo.

Schubring, G. (1987). On the methodology of analysing historical textbooks: Lacroix as textbook author. For the Learning of Mathematics, 7(3), 41-51.

Shen, K., Crossley, J. N. y Lun, A. (1999). The Nine Chapters on the Mathematical Art: Companion and Commentary. Oxford, Reino Unido: Oxford University Press. 
Swetz, F. J. (2014) Expediciones matemáticas. La aventura de los problemas matemáticos a través de la historia (José Migual Parra, trad.). Madrid, España: La esfera de los libros.

Tosca, T. V. (1757). Compendio matemático (Tomo II). Valencia, España: Imprenta de Joseph García.

Vallejo, J. M. (1841). Tratado elemental de Matemáticas escrito de orden de $S$. $M$. para uso de los caballeros seminaristas del seminario de nobles de Madrid y demás casas de educación del Reino (Cuarta edición). Madrid, España: Imp Garrayasaza.

Vallín, A. (1862). Elementos de Matemáticas. Madrid, España: Imprenta de Santiago Aguado.

Ventallol, J. (1612). Practica mercantivol (J. B. Tolrá, trad.; original 1521). Tarragona, España: Gabriel Roberto.

Bernardo Gómez Alfonso

Universidad de Valencia

b.gomez@uv.es 